\title{
Hepatic steatosis associated with decreased $\beta$-oxidation and mitochondrial function contributes to cell damage in obese mice after thermal injury
}

\author{
Li Diao', Christopher Auger ${ }^{1}$, Hisato Konoeda ${ }^{1}$, Ali-Reza Sadri ${ }^{1}$, Saeid Amini-Nik $\mathbb{1}^{1,2}$ and Marc G. Jeschke ${ }^{1,2,3}$
}

\begin{abstract}
Severely burned patients who are morbidly obese have poor clinical outcomes with aggravated metabolic consequences, a higher incidence of multiple organ dysfunction/failure, and significantly increased morbidity and mortality. The underlying mechanisms of these adverse outcomes are essentially unknown. Since the liver is one of the central metabolic organs, we hypothesized that thermal injury in obese patients leads to substantially increased lipolysis, hepatic fat infiltration, resulting in profound hepatic cellular and organellar alterations, consequently causing liver damage and severely augmented metabolic dysfunction. We tested this hypothesis using an obese mouse model subjected to a $20 \%$ total body surface area burn injury. C57BL/6 mice were randomly divided into low-fat diet (LFD) and high-fat diet (HFD) sham and burn groups ( $n=6$ per group) and fed for 16 weeks. 7 days after the thermal injury portal and cardiac blood were taken separately and liver tissue was collected for western blotting and immunohistochemical analysis. Gross examination of the liver showed apparent lipid infiltration in HFD fed and burned mice. We confirmed that augmented ER stress and inhibition of Akt-mTOR signaling dysregulated calcium homeostasis, contributed to the decrease of ER-mitochondria contact, and reduced mitochondrial $\beta$-oxidation in HFD fed and burned mice, leading to profound hepatic fat infiltration and substantial liver damage, hence increased morbidity and mortality. We conclude that obesity contributes to hepatic fat infiltration by suppressing $\beta$-oxidation, inducing cell damage and subsequent organ dysfunction after injury.
\end{abstract}

\section{Introduction}

Modern civilization features redundant access to food supply and thus excessive caloric intake, which is the leading cause of pandemic obesity ${ }^{1}$. Consequently, clinicians are seeing more and more obese patients than ever in history. Even though there is an increased incidence of certain comorbid health problems (such as diabetes, hypertension, sleeping dyspnea) in obese people, their

\footnotetext{
Correspondence: Marc G. Jeschke (marc.jeschke@sunnybrook.ca)

${ }^{1}$ Sunnybrook Research Institute, Toronto, ON, Canada

${ }^{2}$ Department of Surgery, Division of Plastic Surgery, Division of General

Surgery, Department of Immunology, University of Toronto, Toronto, ON,

Canada

Full list of author information is available at the end of the article

Edited by C. Munoz-Pinedo
}

health is generally unaffected unless challenged by additional insults such as trauma, infection, etc. Indeed, multiple clinical studies have shown higher morbidity and mortality rates in obese patients upon acute injury or sickness, although the underlying mechanisms are largely unclear $^{2-4}$. Compared to the wealth of knowledge of metabolic derangements in either obesity (i.e., diabetes) ${ }^{5}$ or post-trauma ${ }^{6}$, there is a dearth of literature concerning the pathology of severe trauma in the obese population. More interestingly, our previous clinical observations showed that mild obesity is beneficial, whereas morbid obesity is detrimental to trauma victims ${ }^{7}$. Hence, there is a fascinating conflict in terms of what is different about mild vs. morbid obesity. We therefore asked what the

\section{(c) The Author(s) 2018}

(c) (i) Open Access This article is licensed under a Creative Commons Attribution 4.0 International License, which permits use, sharing, adaptation, distribution and reproduction cc) in any medium or format, as long as you give appropriate credit to the original author(s) and the source, provide a link to the Creative Commons license, and indicate if changes were made. The images or other third party material in this article are included in the article's Creative Commons license, unless indicated otherwise in a credit line to the material. If material is not included in the article's Creative Commons license and your intended use is not permitted by statutory regulation or exceeds the permitted use, you will need to obtain permission directly from the copyright holder. To view a copy of this license, visit http://creativecommons.org/licenses/by/4.0/. 
underlying mechanisms by which severe obesity worsens clinical outcomes are.

Since the liver is the central metabolic organ, we hypothesized that the augmented effect of chronic hepatic stress as a result of obesity ${ }^{8-10}$ in tandem with the acute perturbation of homeostasis post-trauma ${ }^{11,12}$ would contribute to worse clinical outcomes in this group of patients. We sought to test this hypothesis using a mouse model of high-fat diet (HFD)-induced morbid obesity plus a major burn covering $20 \%$ of the total body surface area (TBSA).

\section{Results}

\section{HFD and burn leads to hepatic fat infiltration and increased lipolysis}

On the basis of well-accepted murine model of HFDinduced obesity ${ }^{13}$, we fed the mice with either HFD or low-fat diet (LFD) for 16 weeks and we observed significantly higher body weight gain in the HFD group vs. the LFD group by the end of the 16 weeks of feeding (Fig. 1a). Concomitant elevation of blood glucose level was observed in HFD mice (Fig. 1b, $p<0.05$ ). We also conducted an intraperitoneal glucose tolerance test
(IPGTT) and found the impaired glucose clearance in HFD mice (Fig. 1c) confirming metabolic alterations.

We then applied a thermal injury of $20 \% \mathrm{TBSA}^{14}$ by the end of the 17th week after the initiation of the feeding. We ended the study 6 days after the thermal injury and conducted another IPGTT, which showed impaired blood glucose clearance at $30 \mathrm{~min}$ and $1 \mathrm{~h}$ after the intraperitoneal injection of dextran (Fig. 1d, e). These IPGTT results indicate increased insulin resistance in the obese mice both in sham and post-burn.

Gross examination of the liver at the end of the study revealed some yellow/pink discoloration of the liver in HFD sham and LFD burn animals. This color change was even more pronounced in the HFD burned mice (Fig. 1f), indicating increased hepatic fat infiltration; this finding was confirmed by H\&E staining, which clearly shows increased fat infiltration of the liver in HFD or burned animals, with the greatest fat infiltration in HFD burned animals (Fig. 1g).

We hypothesized that increased hepatic fat infiltration is due to increased levels of circulating free fatty acids (FFA). When comparing plasma levels of FFA, we observed elevated FFA in HFD vs. LFD mice regardless of

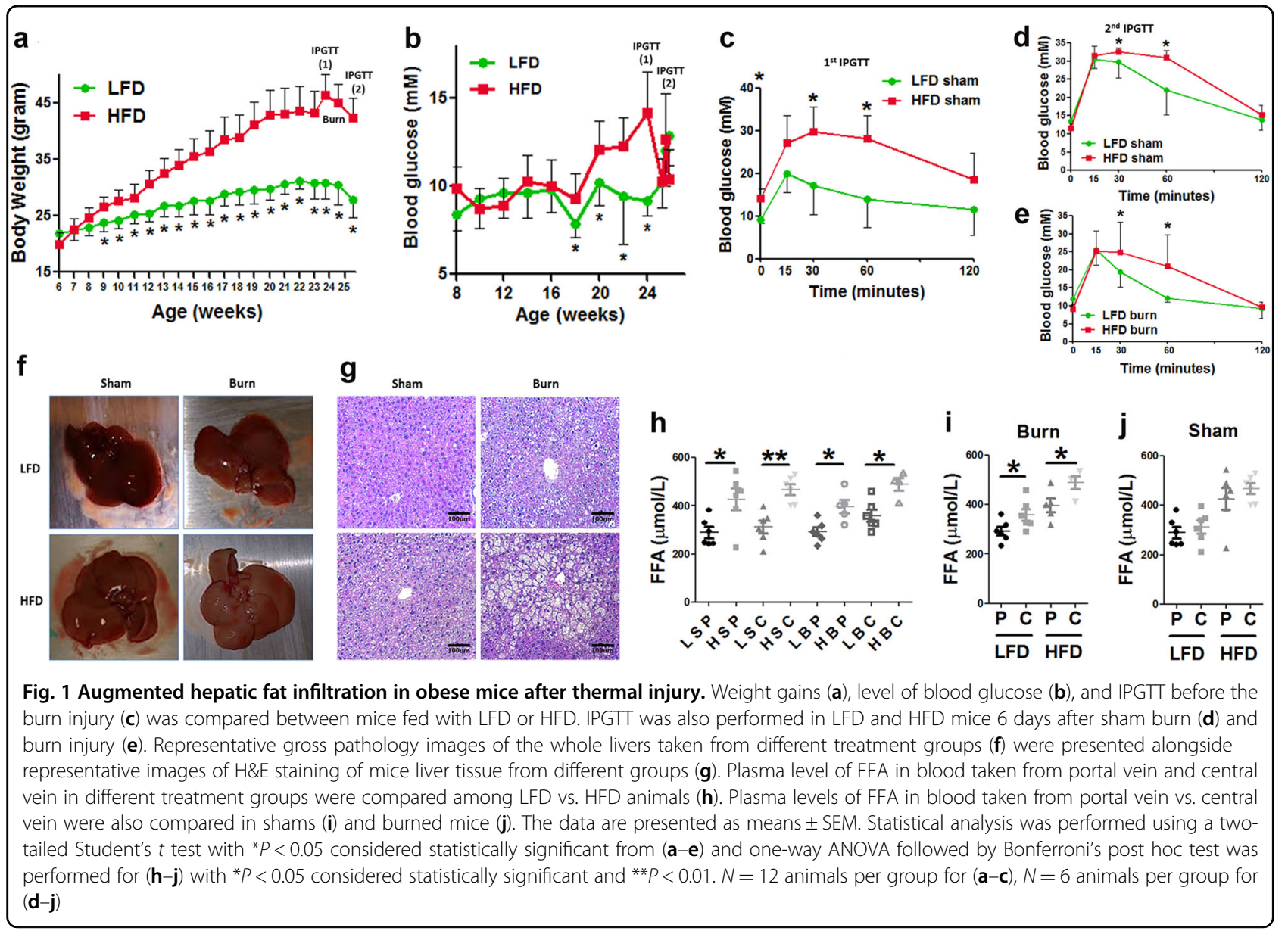


the comparison between portal and central vein blood samples or between shams and scald burned animals (Fig. 1h, $p<0.05$ ). More importantly, we observed significantly higher level of FFA in central vein blood than that in portal vein blood in burned mice (Fig. 1i, $p<0.05$ ) but not in shams (Fig. 1j). Such significantly elevated FFA in central vein blood indicates increased lipolysis of the peripheral adipose tissue after thermal injury ${ }^{15}$. These observations indicated that a HFD followed by a burn injury is associated with increased lipolysis and circulating FFA, resulting in an increased pre-load of lipids to the liver contributing to substantial hepatic fat infiltration.

\section{De novo lipogenesis is not activated in HFD mice after thermal injury}

To examine how increased FFA pre-load was associated with fat infiltration of the liver, we hypothesized and determined if there was an increase in de novo lipogenesis commonly considered as the significant source of intrahepatocellular lipids in fatty liver diseases ${ }^{16}$. Western blot analysis showed the level of liver tissue inhibitory phospho-acetyl CoA carboxylase (Ser79) (p-ACC) was significantly decreased in HFD-fed mice with or without burn injury as compared with LFD sham (Fig. 2a, b, $p<0.05)$, implicating increased conversion from acetylCoA to malonyl-CoA in HFD-fed mice. However, the level of hepatic fatty acid synthase (FASN), the key ratelimiting enzyme of de novo lipogenesis ${ }^{17}$, was significantly decreased in HFD fed mice, especially in HFD burned group (Fig. $2 \mathrm{e}, \mathrm{f}, p<0.01$ ), indicating that there is no activation of lipogenesis albeit the possible increased substrate pressure of malonyl-CoA. There were no significant changes in liver tissue level of p-ACC and FASN in LFD burned mice as compared with LFD shams, indicating that burn alone has no significant impact on hepatic lipogenesis. To further confirm this finding, we performed immunofluorescent staining of p-ACC and FASN in the liver tissue sections (Fig. 2c, g). Statistical analysis of the positive cell counts demonstrated results consistent with the Western blot analyses (Fig. 2d, h). Hence, despite the increased FFA pre-load, there is no evidence supporting the significant increase in the de novo lipogenesis in the liver tissue after HFD and/or thermal injury.

\section{Decreased hepatic lipid $\beta$-oxidation and attenuated mitochondrial electron transport chain (ETC) function associated with hepatic fat infiltration}

To investigate the mechanisms of the increased hepatic fat infiltration, we asked next whether it can be attributed to reduced lipid oxidation. We measured the expression of hepatic carnitine palmitoyltransferase-1 (CPT1A), which is the rate-limiting enzyme for liver mitochondrial $\beta$-oxidation, translocating fatty acids across the mitochondrial membrane. The level of the expression of CPT1A would thus reflect the hepatic $\beta$-oxidation activity $^{18}$.

Western blot analysis showed increased expression of CPT1A in HFD shams and LFD burned mice as compared with LFD sham (Fig. 3a, b, $p<0.01$ ), but not in HFD burn animals. Such an increase in CPT1A in HFD sham and LFD burn is consistent with other studies showing that hepatic mitochondrial $\beta$-oxidation is enhanced in the liver of genetically obese (ob/ob) mice ${ }^{19}$, HFD-fed rats ${ }^{20}$ as well as in patients with steatohepatitis ${ }^{21}$, implicating the increased substrate pressure and activation of the compensatory mechanisms of lipid turnover such as hepatic peroxisome proliferator-activated receptor alpha $($ PPAR $\alpha)$. However, there was a significantly lower level of CPT1A in HFD burned animals as compared with that of HFD only or burn only group. It is interesting to note the divergent response of lipid metabolism between HFD sham and HFD burned animals. While there is likely an increase in malonyl-CoA in both groups due to the activation of $A C C$, it seems that in HFD sham mice, significantly activated lipid $\beta$-oxidation might be compensatory enough to limit the magnitude of the fat infiltration, whereas in HFD burned mice, the accumulation of the malonyl-CoA might be overwhelming and significantly inhibit $\beta$-oxidation, contributing to augmented hepatic fat infiltration ${ }^{22}$.

To confirm our western blotting data, we performed immunofluorescent staining of CPT1A in liver tissue sections. We noticed stronger positive signals of CPT1A in HFD shams and LFD burned mice as compared with LFD shams (Fig. 3c) and statistical analysis of the positive cell counts demonstrated results that are consistent with western blot analyses (Fig. 3d). This suggests that in HFD burned animals, the liver is not able to $\beta$-oxidize the significantly increased inflow of FFA from peripheral lipolysis, resulting in hepatic accumulation of FFA.

To further investigate the underlying mechanisms of impaired hepatic lipid metabolism in HFD burned mice, we analyzed the mitochondrial ETC activities in line with the changes in the hepatic mitochondrial lipid $\beta$-oxidation ${ }^{23}$. The increase of complex I and complex III activity in HFD groups implies that there may be an increase in ROS production as these are the primary sites of superoxide formation. Moreover, the lowered activity of ATP synthase in the HFD burned group is particularly detrimental, as it suggests impaired energy formation in this cohort of mice (Fig. $3 e-j)^{24,25}$. Furthermore, a significant decrease of the Complex II activity not only reinforced the likelihood of impaired mitochondrial energy production and increased ROS production, but also implicated cell damage in HFD burned animals ${ }^{26}$. 
a

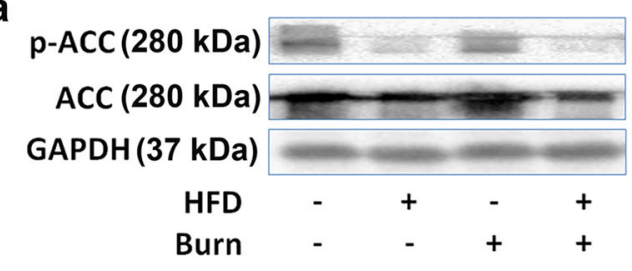

b

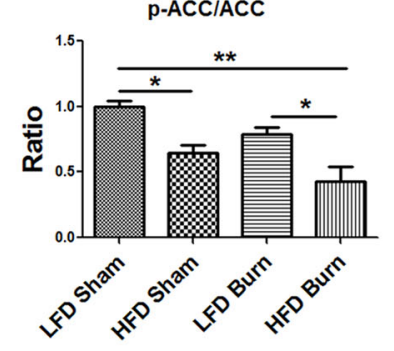

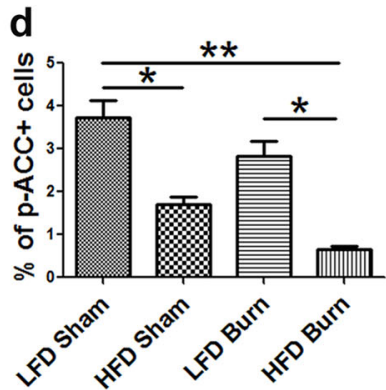

C

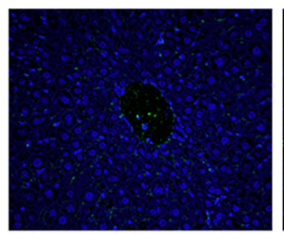

negative control

e

FASN (273 kDa)

GAPDH (37 kDa)

BFD

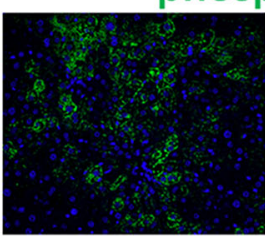

LFD, Sham

HFD, Sham

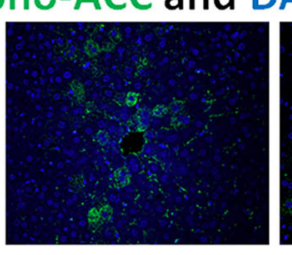

f

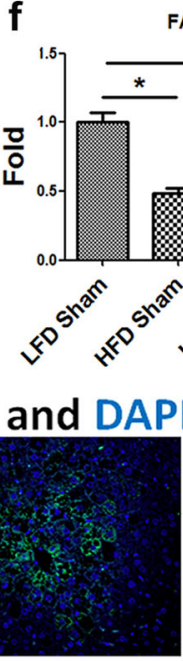

FASN and DAPI
HFD, Sham

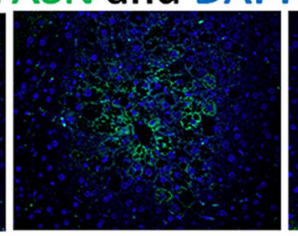

minstam

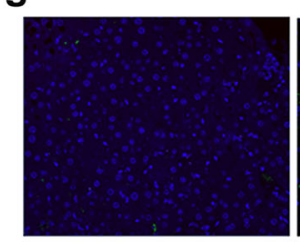

negative control

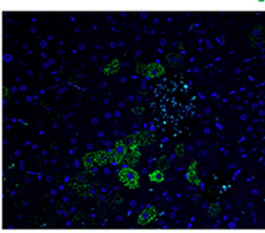

LFD, Sham

Fig. 2 Repression of de novo lipogenesis in HFD mice after thermal injury. Representative images (a) and quantitative densitometric analyses (b) of the Western blot of phospho-ACC (Ser79) and ACC were presented alongside immunofluorescent staining of phospho-ACC (c, magnification $\times 200$ ) and percentage of phospho-ACC positive cells (d) in liver tissue. Representative images (e) and quantitative densitometric analyses (f) of the western blot of FASN were presented alongside immunofluorescent staining of FASN ( $\mathbf{g}$, magnification $\times 200$ ) and percentage of FASN positive cells (h) in liver tissue. Data are presented as means \pm SEM. ${ }^{*} P<0.05$ and ${ }^{* *} P<0.01 . N=6$ animals per group

Perturbed inter-organelle $\mathrm{Ca}^{2+}$ homeostasis correlated with decreased ER-mitochondrial contact

Considering the importance of $\mathrm{Ca}^{2+}$ homeostasis in mitochondrial bioenergetics, we sought to investigate whether there were perturbation in ER-mitochondrial $\mathrm{Ca}^{2+}$ homeostasis in HFD and/or burned mice as compared with LFD sham by checking several key regulators of mitochondrial $\mathrm{Ca}^{2+}$ channels, including inositol 1,4,5-triphosphate receptor 1 (IP3R1), IP3R3, Voltagedependent anion channel 1 (VDAC1), p-Akt (Fig. 4a) ${ }^{27,28}$.
While there were no significant changes in IP3R1 among the groups (Fig. 4b, $p>0.05$ ), there was a significant decrease of IP3R3 levels in burned mice as compared with LFD sham (Fig. 4c, $p<0.05$ ). Since IP3R3 is the major channel for $\mathrm{Ca}^{2+}$ efflux from ER to mitochondria under mild stress conditions ${ }^{29}$, decreased IP3R3 implicated lower $\mathrm{Ca}^{2+}$ levels in mitochondria due to insufficient $\mathrm{Ca}^{2}$ + influx from the ER. Also, considering that VDAC1 is a multi-functional channel involved in $\mathrm{Ca}^{2+}$ and metabolite transport, energy production and in ER-mitochondria 


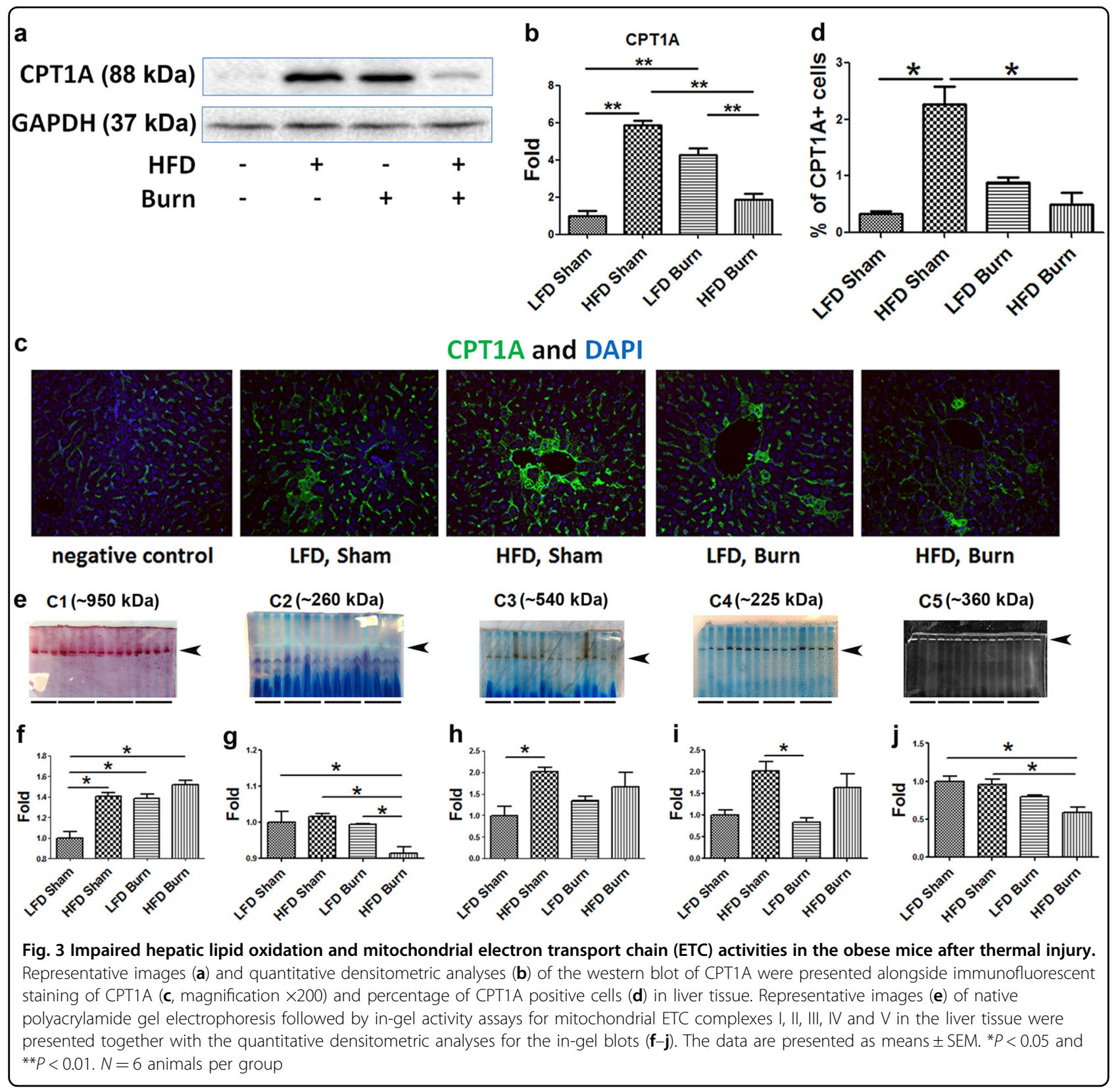

structural and functional association ${ }^{30}$, significantly decreased VDAC1 in HFD sham and LFD burned mice not only indicated the possibility of decrease in mitochondrial mass but also implicated the derangement of $\mathrm{Ca}^{2+}$ transport between ER and mitochondria (Fig. 4d, $p<0.01$ ). Nevertheless, in HFD burned mice, significantly decreased phospho-Akt (Ser473) and Rictor (Fig. 4e, f, $p<0.05$ ) indicated impairment of AktmTORC2 signaling, while it has been well accepted that inhibition of Akt-mTORC2 signaling and subsequent phosphorylation of IP3R in general account for the depletion of $\mathrm{Ca}^{2+}$ from ER to mitochondria, thus triggering cell death pathways ${ }^{31}$.
More importantly, with the growing appreciation of the ER-mitochondrial axis, we postulated that a decrease of ER-mitochondria contact in HFD and/or burned mice would contribute to the aforementioned perturbed inter-organelle $\mathrm{Ca}^{2+}$ homeostasis and mitochondrial energy production. Since mitofusin 2 (MFN2) is widely accepted as the major regulator of the mitochondria-ER contact ${ }^{32,33}$, and its coupling molecule, OPA1 is the key regulator of mitochondrial inner membrane fusion and cristae structuring ${ }^{34}$, we performed western blot analysis on the two proteins (Fig. 4g). A significant decrease of MFN2 in burned mice indicated decreased ER-mitochondrial contact after 


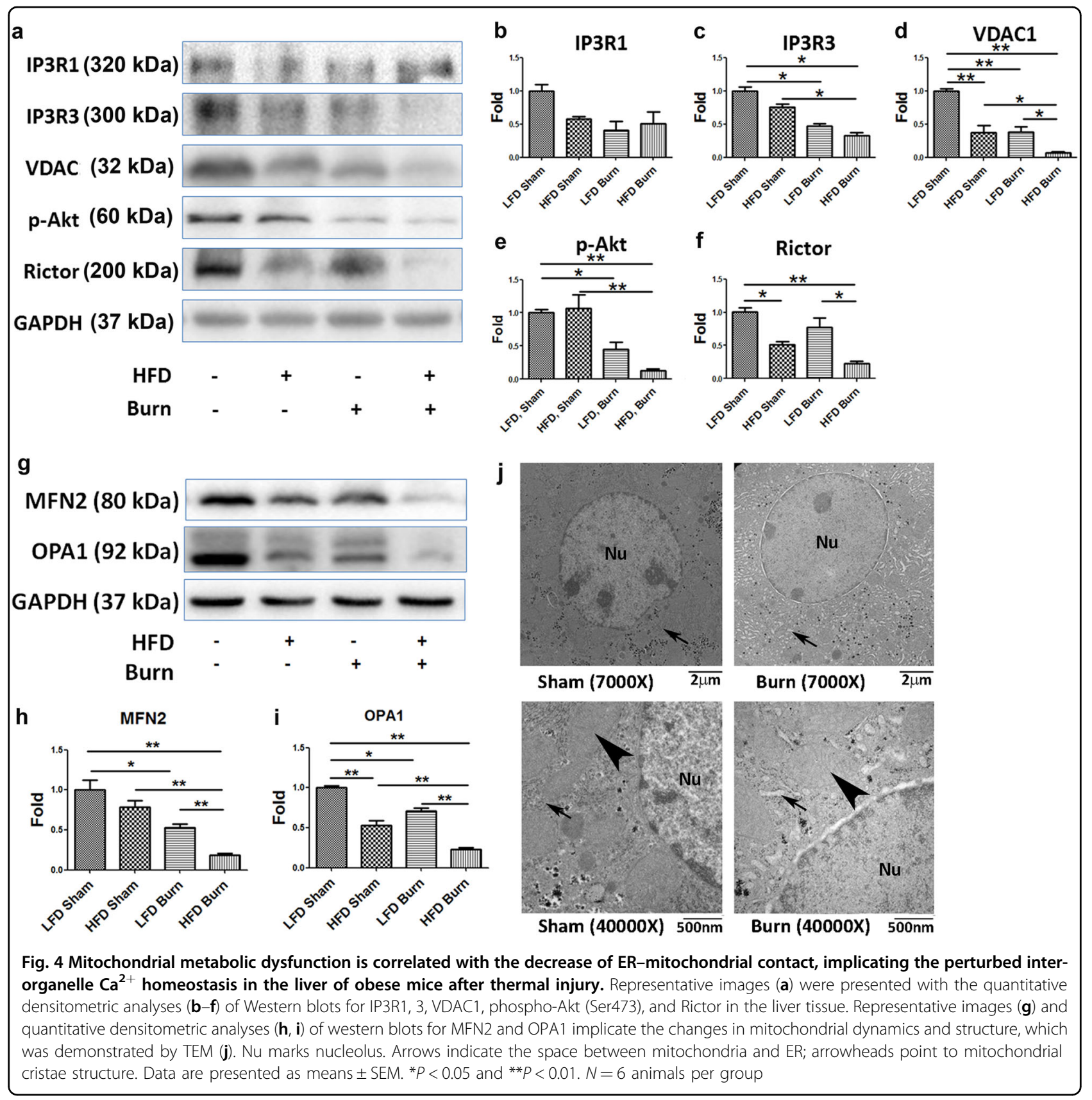

burn injury. A further decrease of ER-mitochondrial contact was implicated in HFD burned mice as compared with either HFD sham or LFD burn (Fig. 4h, $p<0.01)$. A similar pattern was noted for OPA1 levels (Fig. 4i, $p<0.01$ ). These findings in western blot analysis were confirmed via transmission electron microscopy (TEM) (Fig. 4j). As compared with sham, increased space among the ER and mitochondria (arrows) as well as the shrinkage of the mitochondrial cristae (arrowheads) were evident in the liver tissue sections of the burned mice.
Augmented hepatic ER stress, inflammasome activation and aggravated cell damage in HFD mice after thermal injury

With the above evidence of the changes in ER-mitochondrial structure, function and contact, it was reasonable to further postulate a concomitant cellular stress response in HFD and/or burned animals ${ }^{35}$. We thus determined the level of several important hepatic ER stress and subsequent unfolded protein responses (UPR) markers $^{36}$. western blots (Fig. 5a) and densitometric analysis demonstrated augmented ER stress in the liver of 


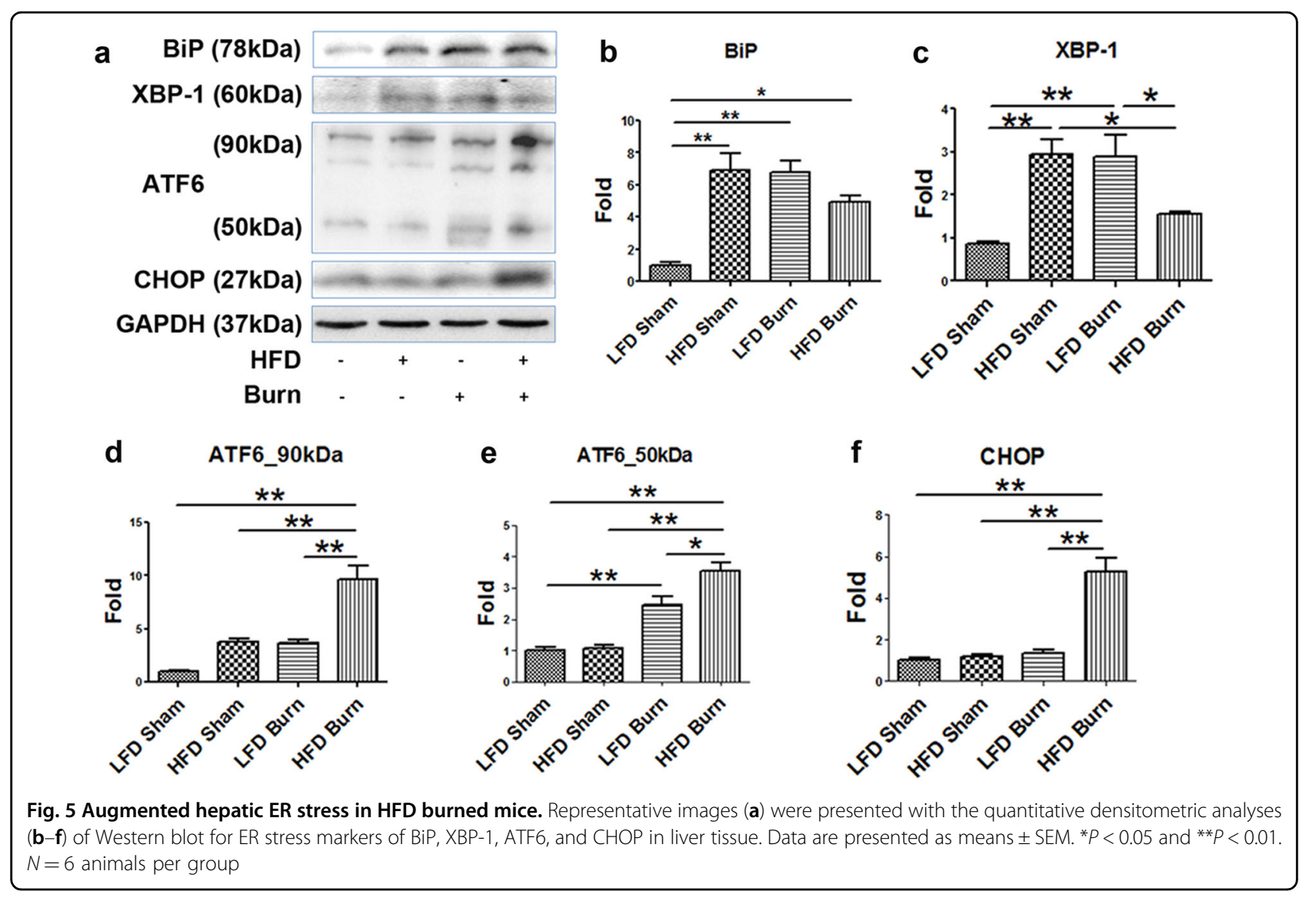

HFD and/or burned animals. We observed the following two patterns of UPR upon the HFD and/or burn injury: (1) the significant activation of hepatic BiP and XBP-1 in either HFD sham or LFD burn group $(p<0.01)$, whereas less significant (BiP) or no significant change (XBP-1) in HFD plus burn group as compared with LFD sham control (Fig. 5b, c); (2) the level of ATF6 and CHOP increased significantly in HFD plus burn group (Fig. $5 \mathrm{~d}-\mathrm{f}, p<0.01$ ). Considering that $\mathrm{CHOP}$ is a pro-apoptotic transcription factor, we then sought for the evidence of cell damage upon HFD and/or thermal injury ${ }^{37}$. TUNEL staining of liver tissue sections confirmed the aggravated apoptosis in HFD sham, LFD burn and HFD burn groups as compared with LFD sham (Fig. 6a). Interestingly, we noticed that hepatocyte apoptosis was more severe in burned mice (Fig. 6b, sham vs. burn, $p<0.01$ ), whereas stromal cell apoptosis was more significant in HFD treatment (Fig. 6c, LFD vs. HFD, $p<0.01)$. Nevertheless, a significantly higher rate of apoptosis was seen in HFD burned mice when compared to LFD shams and either intervention of HFD or burn alone $(p<0.01)$.

We postulated the increased inflammasome activation that contributes to the apoptosis of the stromal cells ${ }^{38}$. This was confirmed by the western blot of NOD-like receptor, pyrin domain containing 3 (NLRP3) and
Caspase 1 (both total and cleaved form) in the liver tissue (Fig. 6d-g, $p<0.01$ ). Also, elevated levels of cleaved Caspase 3 and Poly ADP ribose polymerase (PARP) were also consistent with the TUNEL staining, demonstrating the aggravated cell damage particularly in HFD burned mice (Fig. 6h, $p<0.01$; 6i, $p<0.05$ ).

\section{Discussion}

In the current study, we aimed to determine why patients with obesity have altered metabolism and decreased survival after burn. We focused on liver pathology and used a mouse model of HFD-induced morbid obesity and 20\% TBSA scald burn. The thermal injury model is well described, but we ensured adequate hypermetabolic and inflammatory responses when combining it with a high-fat diet-induced obesity.

In this model, we found profound hepatic fat infiltration in HFD burned mice, which is mainly attributable to increased lipolysis and impaired hepatic lipid $\beta$-oxidation and correlates with liver damage. It is very interesting to note that increased hepatic infiltration is not due to increase de novo lipogenesis. To investigate the underlying mechanisms of such metabolic impairment and tissue damage, we compared the hepatic ER stress responses, changes in mitochondrial ETC activities, 


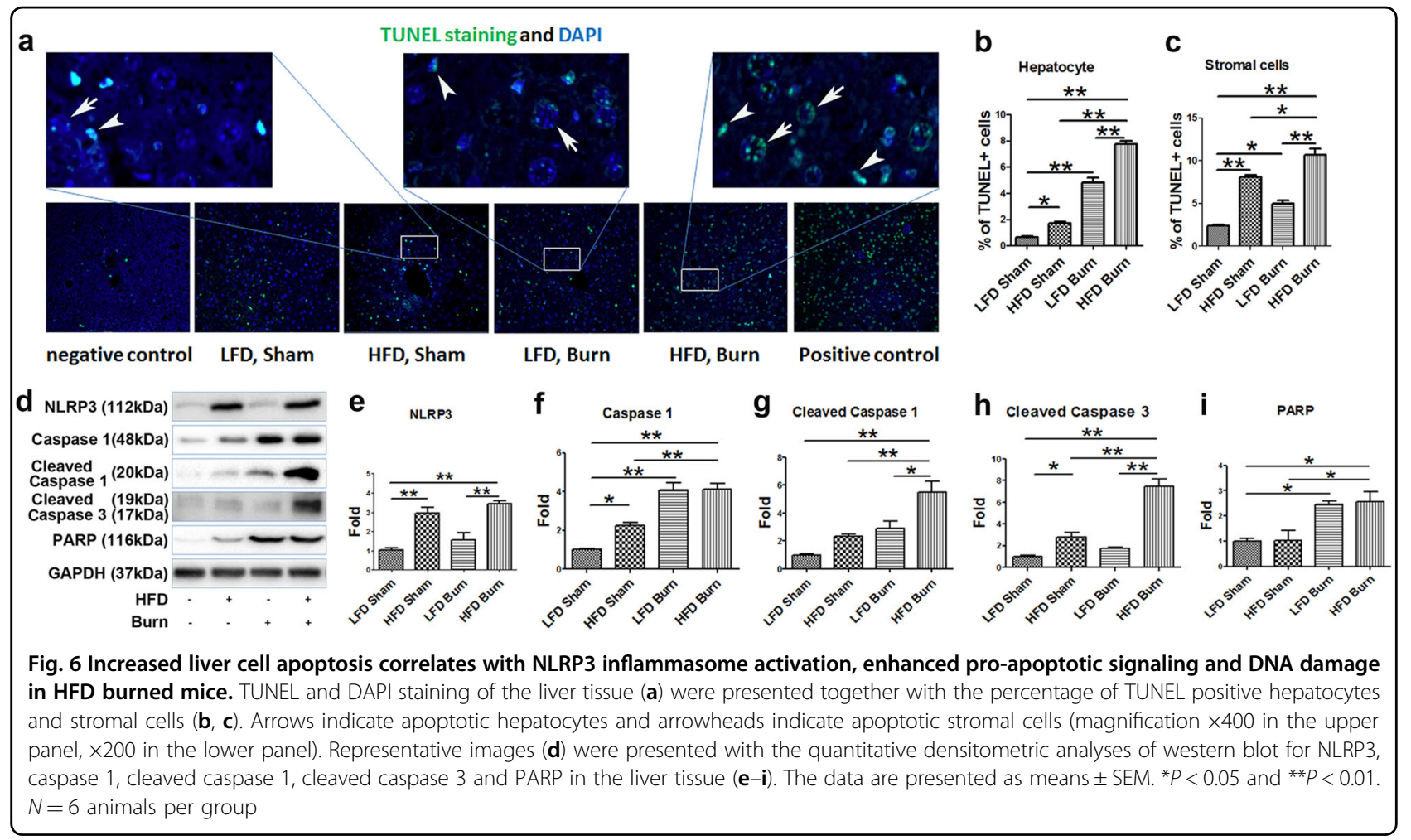

ER-mitochondria communication, inflammasome activation and apoptosis signaling among sham, burn and HFD plus burn groups. We noticed that with a burn injury alone the hepatic responses are generally including the activation of ER UPR of increased expression of BiP and XPB-1 ${ }^{39,40}$, and higher levels of CPT1A for increased $\beta$-oxidation. Mitochondrial ETC activities and ATP synthesis are also well maintained and inflammasome activation and apoptosis are mildly increased. Nevertheless, at least 2 phenomena implicated metabolic derangement after burn injury as compared with sham animals: 1) decreased mitochondria mass and perturbation of mitochondrial $\mathrm{Ca}^{2+}$ homeostasis as is indicated by the changes in IP3R3 and VDAC1; 2) decreased ER-mitochondria contact and mitochondrial dynamics as is manifested by the changes in the levels of MFN2 and OPA1 and morphological alterations seen in TEM. With the onset of ER stress, to chaperone the increased nascent, unfolded or misfolded proteins, BiP tends to dissociate from IP3R1 which is the major channel of $\mathrm{Ca}^{2+}$ flux from ER to mitochondria under physiological conditions, leading to inhibition of the IP3R1 $\mathrm{Ca}^{2+}$ channel. Moreover, a significant decrease of the IP3R3 level as seen in burned animals may also have an impact by decreasing mitochondrial $\mathrm{Ca}^{2+}$ levels. This is consistent with the decreased ATP synthase activity, decreased ER-mitochondria contact, and therefore, decreased energy production and, possibly concomitant increase in
ROS production ${ }^{41}$. Furthermore, derangement of mitochondrial dynamics as was manifested by the changes in MFN2 and OPA1 links impaired energy production with cell damage ${ }^{34}$.

In HFD-induced obese mice after burn injury, drastic changes in almost every aforementioned cellular process brought about a significantly different outcome as compared with burn injury alone. Augmented ER stress was beyond the cellular capacity of molecular chaperoning and pro-apoptotic signaling was activated as was indicated by significantly increased levels of CHOP. In contrast to the inhibition of $\mathrm{Ca}^{2+}$ efflux from hepatic ER to mitochondria in LFD burned mice, there might be dysfunctional $\mathrm{Ca}^{2+}$ transport from ER to mitochondria due to significant activation of IP3R which is termed IP3-induced $\mathrm{Ca}^{2+}$ release ${ }^{42}$. We hypothesize that increased mitochondrial $\mathrm{Ca}^{2+}$ levels contribute to further impairment of energy production and aggravated cell damage ${ }^{43}$. Importantly, a greater decrease of ER-mitochondrial communication in HFD burned mice was seen with the concomitant and significant suppression of OPA1 and inhibition of mitochondrial ETC complexes activities, indicating impairment of mitochondrial energy production, and ultimately, aggravated cell damage ${ }^{44}$.

In summary, in LFD mice, burn injury stimulates an ER stress response with the increased likelihood of a lower level of mitochondrial $\mathrm{Ca}^{2+}$ and subsequent decreased ATP and increased ROS production, as well as decreased 


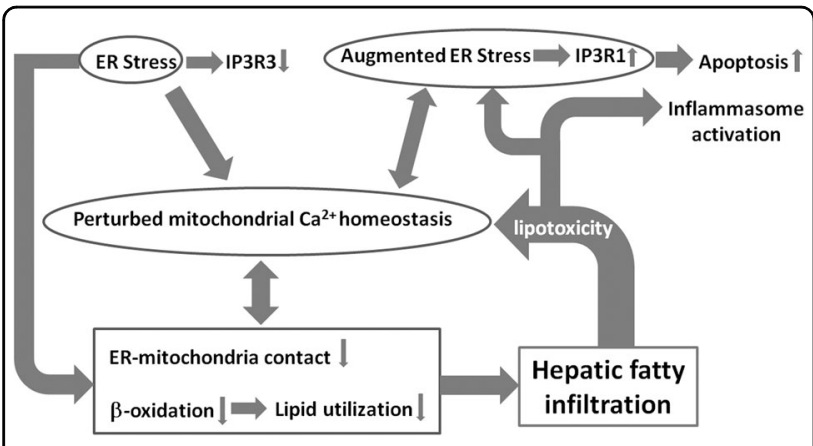

Fig. 7 Hepatic fat infiltration is attributable to the vicious cycle of ER stress, mitochondrial dysregulation and cell damage in HFD burned mice. Hepatic ER stress and disturbed ER-mitochondria communication lead to the derangement of mitochondrial ETC activities, energy production, and impaired lipid metabolism. This contributes to the increased hepatic fat infiltration, which, together with hepatic inflammasome activation, results in increased liver damage in HFD burned mice

ER-mitochondrial contact, all correlated with a mild lipid infiltration. In HFD shams, there is mitochondrial fragmentation and lipid accumulation in the liver albeit compensatory increase of lipid $\beta$-oxidation. This is accompanied with NLRP3 inflammasome activation and increased apoptosis as compared with LFD shams. When HFD mice were challenged by burn injury, augmented ER stress-induced pro-apoptotic signaling; mitochondrial energy production was significantly impaired with further perturbed $\mathrm{Ca}^{2+}$ homeostasis; uncompensated impairment of ER-mitochondrial contact, faulty $\beta$-oxidation, and inflammasome activation occurred, leading to significantly decreased lipid turnover. Subsequent fat infiltration would aggravate ER stress, forming a vicious cycle and leading to liver organ damage (Fig. 7). As such, in animals with obesity and severe burn, it is the impairment of multiple cellular processes which compound upon each other that deregulates lipid homeostasis and, as a consequence, worsens outcomes for obese trauma victims. Accordingly, early and effective interventions to attenuate ER stress, inflammasome activation, and ROS production, as well as treatment to stimulate mitochondrial dynamics and restore mitochondrial $\mathrm{Ca}^{2+}$ homeostasis would be beneficial to this group of patients.

\section{Materials and Methods}

\section{Animal model}

Animal experiments were approved by the Animal Care and Use Committee of Sunnybrook Research Institute (AUP \#467) in Toronto, ON. The National Institutes of Health Guidelines for the Care and Use of Experimental Animals were met. 6-week-old male C57BL/6 mice were purchased from The Jackson Laboratory (ME, USA) and were randomly chosen to receive HFD (TD.06414,
Harlan Laboratories, WI, USA) to induce obesity; mice were fed low-fat diet (LFD, TD.08806, Harlan Laboratories, WI, USA) as control. After 16 weeks of feeding, IPGTT was performed by intraperitoneal injection of $20 \%$ glucose solution ( $2 \mathrm{~g}$ glucose per $\mathrm{kg}$ body weight) after overnight fasting followed by blood glucose measurement (Accu-Chek test strips, Roche, USA) at 0, 15, 30,60 and $120 \mathrm{~min}$ after the glucose intraperitoneal injection. The animals in each group were sub-divided into sham and burned groups ( $N=6$ in each group). HFD/LFD and water was given ad libitum upon arrival until sacrifice. The animals were randomized into 4 groups: LFD sham, HFD sham, LFD burn, and HFD burn. A well-established method was used to induce a fullthickness scald burn of $20 \%$ TBSA $^{45}$. Second IPGTT was performed on post-burn day 6 and all the animals were sacrificed on post-burn day 7 .

\section{Plasma and tissue collection}

Blood was collected from portal vein and cardiac puncture, respectively. Blood and liver tissue were processed as previously described ${ }^{15}$.

\section{Western blotting}

Antibodies against p-ACC (Ser79), ACC, FASN, CPT1A, IP3R1, VDAC, phospho-Akt (Ser473), Rictor, MFN2, BiP, CHOP, Caspase-1, cleaved Caspase-1, cleaved Caspase-3, PARP, and GAPDH were purchased from Cell Signaling (Danvers, MA, USA). Anti- NLRP3, anti-XBP-1, and anti-ATF6 antibodies were purchased from EMD Millipore (Billerica, MA, USA). Anti-IP3R3 antibody was purchased from BD Biosciences (San Jose, CA, USA). Clarity Western ECL substrate was purchased from BioRad (Hercules, CA, USA). Liver homogenates $(50 \mu \mathrm{g}$ of protein per well) were separated by $10 \%$ SDS-PAGE gel, proteins were transferred to nitrocellulose membrane as previously described ${ }^{15}$, and then blots were probed using the antibodies listed above. Band intensities were detected, normalized and quantified with the Chemidoc and Image Lab 5.0 software (Bio-Rad Laboratories, Hercules, CA). GAPDH was used as loading control.

\section{In-gel mitochondrial ETC activity assays}

In-gel mitochondrial ETC activity assays were performed as described previously ${ }^{23,46}$.

\section{Immunofluorescent multi-channel staining of liver}

Antibody staining was performed as described previously ${ }^{15,47}$. Primary antibodies were the same as in Western blotting. The percentage of marker-positive cells was determined by taking representative images and directly counting cell number by blindfolded third party. Cell enumerations for each experiment are listed in the text or figure legends. 


\section{Hematoxylin and eosin (H\&E) staining and transmission electron microscopy (TEM) of tissue sections}

Liver tissue was fixed, sectioned, and stained as described previously ${ }^{15,47}$.

\section{Determination of free fatty acids (FFA), glycerol and triglyceride levels in blood}

Levels of FFA, glycerol and triglyceride in the blood were determined using FFA, glycerol and triglyceride colorimetric assay kits according to the manufacturer's instructions (Cayman Chemical, Ann Arbor, Michigan, USA).

\section{Statistical analysis}

The statistical analysis was performed using Prism version 5.01 (GraphPad Software, San Diego, CA). Oneway ANOVA with Bonferroni's Multiple Comparison Test was used unless otherwise specified and $P<0.05$ was considered statistically significant.

\section{Acknowledgements}

This research was supported by the National Institutes of Health (2R01 GM087285-05A1), Canadian Institutes of Health Research (\#123336), the CFI Leaders Opportunity Fund (\#25407) and a generous donation from Toronto Hydro

\section{Author details}

${ }^{1}$ Sunnybrook Research Institute, Toronto, ON, Canada. ${ }^{2}$ Department of Surgery, Division of Plastic Surgery, Division of General Surgery, Department of Immunology, University of Toronto, Toronto, ON, Canada. ${ }^{3}$ Ross Tilley Burn Centre, Sunnybrook Health Sciences Centre, Toronto, ON, Canada

\section{Authors' contributions}

L.D., S.A.-N., and M.G.J. conceived and designed the experiments; L.D., C.A., H.K and A.-R.S. performed the experiments; L.D., C.A. and H.K. analyzed the data and interpreted the results; S.A.-N. and M.G.J. directed the studies and L.D. wrote the manuscript.

\section{Conflict of interest}

The authors declare that they have no conflict of interest.

\section{Publisher's note}

Springer Nature remains neutral with regard to jurisdictional claims in published maps and institutional affiliations.

Received: 26 September 2017 Revised: 14 February 2018 Accepted: 5 March 2018

Published online: 10 May 2018

\section{References}

1. Ginter, E. \& Simko, V. Type 2 diabetes mellitus, pandemic in 21 st century. Adv. Exp. Med. Biol. 771, 42-50 (2012).

2. Yan, J. et al. Inactivation of bad by ikk inhibits tnfalpha-induced apoptosis independently of nf-kappab activation. Cell 152, 304-315 (2013).

3. Wang, C. Obesity, inflammation, and lung injury (oili): The good. Mediat. Inflamm. 2014, 978463 (2014).

4. Kraft, R. et al. The effect of obesity on adverse outcomes and metabolism in pediatric burn patients. Int. J. Obes. 36, 485-490 (2012).

5. Samuel, V. T. \& Shulman, G. I. The pathogenesis of insulin resistance: Integrating signaling pathways and substrate flux. J. Clin. Invest. 126, 12-22 (2016).
6. Jeschke, M. G. et al. Severe injury is associated with insulin resistance, endoplasmic reticulum stress response, and unfolded protein response. Ann. Surg. 255, 370-378 (2012).

7. Jeschke, M. G. et al. Mild obesity is protective after severe burn injury. Ann Surg. 258, 1119-1129 (2013).

8. Wires, E. S. et al. High fat diet disrupts endoplasmic reticulum calcium homeostasis in the rat liver. J. Hepatol. 67, 1009-1017 (2017).

9. Lionetti, L. et al. High-lard and high-fish-oil diets differ in their effects on function and dynamic behaviour of rat hepatic mitochondria. PLOS ONE 9 e92753 (2014).

10. Mantena, S. K. et al. High fat diet induces dysregulation of hepatic oxygen gradients and mitochondrial function in vivo. Biochem. J. 417, 183-193 (2009).

11. Jeschke, M. G. \& Boehning, D. Endoplasmic reticulum stress and insulin resistance post-trauma: Similarities to type 2 diabetes. J. Cell. Mol. Med. 16, 437-444 (2012).

12. Jeschke, M. G. The hepatic response to thermal injury: Is the liver important for postburn outcomes? Mol. Med. 15, 337-351 (2009).

13. Jiang, T. et al. Diet-induced obesity in c57b//6j mice causes increased renal lipid accumulation and glomerulosclerosis via a sterol regulatory elementbinding protein-1c-dependent pathway. J. Biol. Chem. 280, 32317-32325 (2005).

14. Finnerty, C. C., Przkora, R., Herndon, D. N. \& Jeschke, M. G. Cytokine expression profile over time in burned mice. Cytokine 45, 20-25 (2009).

15. Diao, L. et al. Alternative mechanism for white adipose tissue lipolysis after thermal injury. Mol. Med. 21, 959-968 (2015).

16. Solinas, G., Boren, J. \& Dulloo, A. G. De novo lipogenesis in metabolic homeostasis: More friend than foe? Mol. Metab. 4, 367-377 (2015).

17. Ameer, F., Scandiuzzi, L., Hasnain, S., Kalbacher, H. \& Zaidi, N. De novo lipogenesis in health and disease. Metabolism 63, 895-902 (2014).

18. Lee, K., Kerner, J. \& Hoppel, C. L. Mitochondrial carnitine palmitoyltransferase 1a (cpt1a) is part of an outer membrane fatty acid transfer complex. J. Biol. Chem. 286, 25655-25662 (2011).

19. Brady, L. J., Brady, P. S., Romsos, D. R. \& Hoppel, C. L. Elevated hepatic mitochondrial and peroxisomal oxidative capacities in fed and starved adult obese (ob/ob) mice. Biochem. J. 231, 439-444 (1985)

20. Mollica, M. P. et al. 3,5-diiodo-l-thyronine, by modulating mitochondria functions, reverses hepatic fat accumulation in rats fed a high-fat diet. $J$. Hepatol. 51, 363-370 (2009).

21. Sanyal, A. J. et al. Nonalcoholic steatohepatitis: Association of insulin resistance and mitochondrial abnormalities. Gastroenterology 120, 1183-1192 (2001).

22. Lopez-Vinas, E. et al. Definition by functional and structural analysis of two malonyl-coa sites in carnitine palmitoyltransferase 1a. J. Biol. Chem. 282 18212-18224 (2007)

23. Auger, C., Sivayoganathan, T., Abdullahi, A., Parousis, A. \& Jeschke, M. G. Hepatic mitochondrial bioenergetics in aged $c 57 \mathrm{bl} / 6$ mice exhibit delayed recovery from severe burn injury. Biochim. Biophys. Acta 1863, 2705-2714 (2017).

24. Gusdon, A. M., Song, K. X. \& Qu, S. Nonalcoholic fatty liver disease: pathogenesis and therapeutics from a mitochondria-centric perspective. Oxid. Med. Cell Longev. 2014, 637027 (2014).

25. Murphy, M. P. How mitochondria produce reactive oxygen species. Biochem. J. 417, 1-13 (2009).

26. Tretter, L., Patocs, A. \& Chinopoulos, C. Succinate, an intermediate in metabolism, signal transduction, ros, hypoxia, and tumorigenesis. Biochim. Biophys. Acta 1857, 1086-1101 (2016).

27. Rieusset, J. Endoplasmic reticulum-mitochondria calcium signaling in hepatic metabolic diseases. Biochim. Biophys. Acta 1864, 865-876 (2017).

28. Griffiths, E. J. \& Rutter, G. A. Mitochondrial calcium as a key regulator of mitochondrial atp production in mammalian cells. Biochim. Biophys. Acta 1787, 1324-1333 (2009).

29. Kiviluoto, S. et al. Regulation of inositol 1,4,5-trisphosphate receptors during endoplasmic reticulum stress. Biochim. Biophys. Acta 1833, 1612-1624 (2013).

30. Shoshan-Barmatz, V., De, S. \& Meir, A. The mitochondrial voltage-dependent anion channel 1, $\mathrm{ca}(2+)$ transport, apoptosis, and their regulation. Front. Oncol. 7, 60 (2017).

31. Betz, C. et al. Feature article: Mtor complex 2-akt signaling at mitochondriaassociated endoplasmic reticulum membranes (mam) regulates mitochondrial physiology. Proc. Natl Acad. Sci. USA 110, 12526-12534 (2013).

32. de Brito, O. M. \& Scorrano, L. Mitofusin 2 tethers endoplasmic reticulum to mitochondria. Nature 456, 605-610 (2008).

33. Merkwirth, C. \& Langer, T. Mitofusin 2 builds a bridge between er and mitochondria. Cell 135, 1165-1167 (2008). 
34. MacVicar, T. \& Langer, T. Opa1 processing in cell death and disease-the long and short of it. J. Cell. Sci. 129, 2297-2306 (2016).

35. Shinde, V., Kotla, P., Strang, C. \& Gorbatyuk, M. Unfolded protein responseinduced dysregulation of calcium homeostasis promotes retinal degeneration in rat models of autosomal dominant retinitis pigmentosa. Cell death Dis. 7 e2085 (2016)

36. Huang, $H$. et al. Neuroprotection by eif2alpha-chop inhibition and xbp-1 activation in eae/optic neuritiss. Cell death Dis. 8, e2936 (2017).

37. Szegezdi, E., Logue, S. E., Gorman, A. M. \& Samali, A. Mediators of endoplasmic reticulum stress-induced apoptosis. Embo. Rep. 7, 880-885 (2006).

38. Wen, $H$. et al. Fatty acid-induced nlrp3-asc inflammasome activation interferes with insulin signaling. Nat. Immunol. 12, 408-415 (2011).

39. Ozcan, U. et al. Endoplasmic reticulum stress links obesity, insulin action, and type 2 diabetes. Science 306, 457-461 (2004).

40. Taylor, R. C. \& Dillin, A. Xbp-1 is a cell-nonautonomous regulator of stress resistance and longevity. Cell 153, 1435-1447 (2013).
41. Wang, $Y$. et al. Calcium sensing receptor protects high glucose-induced energy metabolism disorder via blocking gp78-ubiquitin proteasome pathway. Cell death Dis. 8, e2799 (2017).

42. Li, G. et al. Role of ero1-alpha-mediated stimulation of inositol 1,4,5-triphosphate receptor activity in endoplasmic reticulum stress-induced apoptosis. J. Cell. Biol. 186, 783-792 (2009).

43. Hayashi, T. \& Su, T. P. Sigma-1 receptor chaperones at the er-mitochondrion interface regulate $\mathrm{ca}(2+)$ signaling and cell survival. Cell 131, 596-610 (2007).

44. Friedman, J. R. \& Nunnari, J. Mitochondrial form and function. Nature $\mathbf{5 0 5}$ 335-343 (2014).

45. Jeschke, M. G. et al. Insulin protects against hepatic damage postburn. Mol. Med. 17, 516-522 (2011).

46. Wittig, I., Karas, M. \& Schagger, H. High resolution clear native electrophoresis for in-gel functional assays and fluorescence studies of membrane protein complexes. Mol. Cell. Proteom. 6, 1215-1225 (2007).

47. Amini-Nik, S. et al. Beta-catenin-regulated myeloid cell adhesion and migration determine wound healing. J. Clin. Invest. 124, 2599-2610 (2014). 